\title{
Modalities for predicting events in patients with ICDs: Expanding the versatility of FDG PET.
}

\author{
Takashi Kudo, MD, PhD, ${ }^{\mathrm{a}, \mathrm{b}}$ and Arman Erzhanovich Nessipkhan, $\mathrm{MD}^{\mathrm{a}}$ \\ a Department of Radioisotope Medicine, Graduate School of Biomedical Sciences, Nagasaki \\ University, Nagasaki, Japan \\ b Department of Radioisotope Medicine, Atomic Bomb Disease Institute, Nagasaki University, \\ Nagasaki, Japan
}

Received Feb 4, 2021; accepted Feb 4, 2021

doi: $10.1007 / \mathrm{s} 12350-021-02569-y$

\section{See related article, pp. 464-477}

Fluorodeoxyglucose (FDG) and FDG positron emission tomography (PET) was developed in the 1970s, and after nearly 50 years in medical practice, FDG PET is still one of the most versatile imaging modalities in nuclear medicine. The utility of FDG PET is not limited to cardiac diseases, and it has been applied to many other fields, such as oncology, neurology, and even inflammatory and auto-immune diseases. FDG PET was initially used in cardiology to evaluate ischemic heart diseases, such as in viability assessments and the imaging of ischemic damage. It was then expanded to cardiology fields, including cardiac sarcoidosis, large vessel vasculitis, and endocarditis. In this issue of the Journal of Nuclear Cardiology, Jing and colleagues expand the realm of FDG PET to arrhythmogenic diseases ${ }^{1}$. They demonstrated the utility of ECG-gated FDG PET for predicting ventricular arrhythmias in patients with implantable cardioverter defibrillators (ICDs).

Sudden cardiac death (SCD) from ventricular arrhythmia is one of the leading causes of cardiac mortality, accounting for approximately $50 \%$ of all cardiac deaths ${ }^{2}$. The placement of ICDs is a cost-effective primary and secondary prevention measure for $\mathrm{SCD}^{3,4}$. However, a number of issues have yet to be resolved. According to recent guidelines, the most

Reprint requests: Takashi Kudo, MD, PhD, Department of Radioisotope Medicine, Graduate School of Biomedical Sciences, Nagasaki

University, Nagasaki, Japan; tkudo123@nagasaki-u.ac.jp

J Nucl Cardiol 2021;28:478-80.

1071-3581/\$34.00

Copyright (C) 2021 American Society of Nuclear Cardiology. crucial determinant affecting the placement of ICDs is a severely reduced left ventricular ejection fraction (LVEF), which is generally defined as $<35 \%^{2}$. However, the frequency of appropriate firing of ICDs is not so igh $^{5,6}$. It is reported that approximately 10 to $20 \%$ of ICDs are exhibiting inappropriate discharges ${ }^{7,8}$. Although ICDs are a cost-effective treatment, they are still expensive ${ }^{3,4}$. The DANISH trial failed to demonstrate improvements in the survival of patients with nonischemic cardiomyopathy following ICD placement ${ }^{9}$. Although a number of reasons have been proposed to account for this failure, the simple measurement of LVEF is generally considered to be insufficient to identify which patients will benefit from ICD placement. Therefore, more precise methods that provide accurate predictions, which are performed before ICD placement, are needed to select patients who will benefit the most from IDCs ${ }^{10,11}$.

A retrospective study performed by Jing and colleagues measured clinical parameters before ICD placement. They identified the following four parameters closely associated with ventricular arrhythmia: the scar area, phase SD (PSD), bandwidth, and the sum thickening score (STS). Among those four parameters, the scar area is a very common parameter measured without ECG gating. FDG only accumulates in living tissue, and when combined with its good image quality, high resolution, and lack of attenuation artifacts, FDG PET provides the most precise demarcation between living and dead myocardial tissue under chronic conditions. In the acute phase of the disease, such as myocardial infarction and myocarditis, this demarcation is frequently affected by the presence of inflammatory cells, in which FDG accumulates. However, this issue is minimized in the chronic phase, and the size of the truly dead myocardium (not ischemia nor hibernation) can be precisely measured with FDG PET. Thus, FDG PET is 
considered the gold standard for viability assessments of chronic myocardial infarction. By using a combination of perfusion imaging and FDG PET, the myocardium has been divided into the following three categories: Normal (good perfusion with good FDG uptake), damaged but viable (impaired perfusion under hyperemic conditions and/or at rest with the maintenance of FDG uptake), and infarcted/fibrotic (no perfusion at rest with no FDG uptake). The study by Jing and colleagues did not show a relationship between hibernation and events. The ischemic myocardium is known to be a crucial arrhythmogenic substrate, at least under acute conditions. However, discrepancies have been reported for the relationship between ischemic burden and arrhythmogenic events. A previous study by Gupta et al. ${ }^{12}$ showed that the scar burden was associated with major arrhythmic events (MAEs), whereas ischemia was not. In contrast, previous findings by Rijnierse et al. showed that impaired hyperemia was more closely associated with ventricular arrhythmia than with the scar burden ${ }^{13}$. Jing and colleagues did not perform an ischemic analysis. However, when the hibernating myocardium and ischemic myocardium were regarded as one group named "a damaged but viable myocardium", their findings were consistent with Gupta's finding showing no relationship with ischemia. Further studies are needed to clarify whether the remnant ischemic burden is a major contributing factor to MAEs. Nevertheless, the present study supports the scar burden being a major contributor to MAEs after ICD placement that may be precisely measured with FDG PET.

Parameters other than LVEF, such as electrophysiological and imaging parameters, may be useful for risk stratifications. Echocardiography and MRI have frequently been used and proven to be useful ${ }^{14}$. Previous studies indicated that myocardial fibrotic changes measured using echocardiography (strain assessment) and MRI (late gadolinium enhancement: LGE) were related to future MAEs. On the other hand, nuclear medicine was not frequently used to predict MAEs in patients with non-ischemic cardiomyopathy. This may be attributed to its low resolution, which is insufficient for the diffusely distributed patchy changes observed under non-ischemic conditions. Difficulties are associated with assessing minor or thin non-transmural fibrosis using nuclear medicine imaging, even with PET. Jing and colleagues proposed the use of an ECG-gated imaging technique to apply nuclear cardiology in this field. Their results support that dyssynchrony parameters derived from phase analysis and thickening parameters expressed as STS are strongly associated with MAEs. Why those parameters related to MAEs events? A recent study that examined hypertrophic cardiomyopathy revealed a strong correlation between \%LGE and phase parameters on SPECT (bandwidth and PSD) ${ }^{15}$, which may partially explain the present results. Myocardial fibrosis beyond the spatial resolution of nuclear medicine imaging may deteriorate phase parameters, which reflect not only focal but also diffuse damage. By adding a phase analysis to the measurement of scar volumes, nuclear cardiology imaging may provide information on both segmental and diffuse myocardial changes using a single imaging protocol, facilitating assessments before and after ICD placement. Actually, Jing et al. successfully separated the prognoses of patients using a four-point system, giving each parameter (PSD, bandwidth, STS, and scar size) one point.

The present study by Jing and colleagues is not free from some limitations. The sample size was small, and the patient population included those with ischemic and non-ischemic heart diseases. In total, $30 \%$ of the patient population had ischemic cardiomyopathy, which needs to be considered even though it did not strongly influence the results obtained. It is important to note that the prognosis of ischemic cardiomyopathy after ICD placement was shown to be significantly worse than that of non-ischemic cardiomyopathy ${ }^{11}$. The majority of the present results may be applied to non-ischemic cardiomyopathy; however, a separate and larger study limited to non-ischemic disease needs to be performed in order to reach a concrete conclusion. Also, to apply the results to the broad spectrum of patients who may develop MAEs, those with mild left ventricular dysfunction should be studied. The benefits of ICD placement may be more significant in patients with moderate or mild disease than in those with severe disease ${ }^{16,17}$. A recent study showed that ICD placement was more beneficial in asymptomatic (=NYHA I) patients with ischemic cardiomyopathy than in symptomatic patients ${ }^{18}$. A major nuclear cardiology study has not yet been performed on this subset of patients for ICD placement. Based on these findings, structuring a strategy for ICD placement and the prediction of subsequent events needs to be divided into four quadrants with two dimensions: ischemic/non-ischemic and $\mathrm{EF} \geqq 35 \%$ (or $40 \%$ ) $/<35 \%$ (or $40 \%$ ).

Furthermore, the accuracy of a phase analysis using FDG PET remains unclear. A previous study demonstrated that Gated FDG PET could measure dyssynchrony accurately ${ }^{19}$. However, a conflicting study shows only a moderate correlation between phase parameters measured on MPI with Tc-99m labeled agent and that on FDG $\mathrm{PET}^{20}$. In that study, the discrepancy was severe in cases of LV remodeling with a large perfusion defect. This discrepancy in patients with remodeling is a challenging issue, particularly in patients with ischemic cardiomyopathy, because ICD is mainly placed under the condition of a severely reduced 
LV function, which frequently co-exists with $\mathrm{LV}$ remodeling. This discrepancy between Gated FDG PET and Gated MPI may not be due to FDG PET being inferior. Maybe, FDG PET measures phase parameters more precisely than MPI. Considering the excellent image quality of FDG PET and the fact that FDG accumulates in viable myocardium with severely reduced perfusion, it is conceivable that FDG PET can assess the phase parameters more accurately than gated MPI. Since there is currently no gold standard for phase measurements, this debate remains unresolved.

Nevertheless, Jing et al. proved and expanded the utility of FDG PET into the field of arrhythmic diseases. FDG PET is a truly versatile modality for all cardiologists. Fifty-year-old FDG PET is still young, alive and kicking.

\section{References}

1. Jing R, Sun XX, Hua W, Liang C, Yang SW, Hu YR et al. Global and regional cardiac dysfunction quantified by ${ }^{18} \mathrm{~F}$-FDG PET scans can predict ventricular arrhythmia in patients with implantable cardioverter defibrillator. J Nucl Cardiol 2021. Online ahead of print

2. Al-Khatib SM, Stevenson WG, Ackerman MJ, Bryant WJ, Callans DJ, Curtis AB et al (2018) 2017 AHA/ACC/HRS guideline for management of patients with ventricular arrhythmias and the prevention of sudden cardiac death: Executive summary. Heart Rhythm 15:e190-e252

3. Sanders GD, Hlatky MA, Owens DK (2005) Cost-effectiveness of implantable cardioverter-defibrillators. N Engl J Med 353:14711480

4. Holbrook R, Higuera L, Wherry K, Phay D, Hsieh Y-C, Lin K-H et al (2020) Implantable cardioverter defibrillator therapy is cost effective for primary prevention patients in Taiwan: An analysis from the Improve SCA trial. PLoS ONE 15:e0241697

5. Sabbag A, Suleiman M, Laish-Farkash A, Samania N, Kazatsker M, Goldenberg I et al (2015) Contemporary rates of appropriate shock therapy in patients who receive implantable device therapy in a real-world setting: From the Israeli ICD Registry. Heart Rhythm 12:2426-2433

6. Singh JP, Hall WJ, McNitt S, Wang H, Daubert JP, Zareba W et al (2005) Factors influencing appropriate firing of the implanted defibrillator for ventricular tachycardia/fibrillation: Findings from the Multicenter Automatic Defibrillator Implantation Trial II (MADIT-II). J Am Coll Cardiol 46:1712-1720

7. Hofer D, Steffel J, Hürlimann D, Haegeli L, Lüscher TF, Duru F et al (2017) Long-term incidence of inappropriate shocks in patients with implantable cardioverter defibrillators in clinical practice: An underestimated complication? J Interv Card Electrophysiol 50:219-226
8. Bardy GH, Lee KL, Mark DB, Poole JE, Packer DL, Boineau R et al (2005) Amiodarone or an implantable cardioverter-defibrillator for congestive heart failure. N Engl J Med 352:225-237

9. Køber L, Thune JJ, Nielsen JC, Haarbo J, Videbæk L, Korup E et al (2016) Defibrillator implantation in patients with nonischemic systolic heart failure. N Engl J Med 375:1221-1230

10. Bunch TJ. Who Will Benefit From an Implantable Cardioverter Defibrillator? Circulation: Cardiovascular Quality and Outcomes 2019;12.

11. Reeder HT, Shen C, Buxton AE, Haneuse SJ, Kramer DB. Joint shock/death risk prediction model for patients considering implantable cardioverter-defibrillators. Circulation 2019;12.

12. Gupta A, Harrington M, Albert CM, Bajaj NS, Hainer J, Morgan V et al (2018) Myocardial scar but not ischemia is associated with defibrillator shocks and sudden cardiac death in stable patients with reduced left ventricular ejection fraction. JACC Clin Electrophysiol 4:1200-1210

13. Rijnierse MT, de Haan S, Harms HJ, Robbers LF, Wu L, Danad I et al (2014) Impaired hyperemic myocardial blood flow is associated with inducibility of ventricular arrhythmia in ischemic cardiomyopathy. Circ Cardiovasc Imaging 7:20-30

14. Rijnierse MT, Allaart CP, Knaapen P (2016) Principles and techniques of imaging in identifying the substrate of ventricular arrhythmia. J Nucl Cardiol 23:218-234

15. Yuki H, Utsunomiya D, Shiraishi S, Takashio S, Sakamoto F, Tsuda $\mathrm{N}$ et al (2018) Correlation of left ventricular dyssynchrony on gated myocardial perfusion SPECT analysis with extent of late gadolinium enhancement on cardiac magnetic resonance imaging in hypertrophic cardiomyopathy. Heart Vessels 33:623-629

16. Gatzoulis KA, Tsiachris D, Arsenos P, Antoniou C-K, Dilaveris P, Sideris $S$ et al (2019) Arrhythmic risk stratification in post-myocardial infarction patients with preserved ejection fraction: the PRESERVE EF study. Eur Heart J 40:2940-2949

17. Arsenos P, Gatzoulis KA, Doundoulakis I, Dilaveris P, Antoniou CK, Stergios S et al (2020) Arrhythmic risk stratification in heart failure mid-range ejection fraction patients with a non-invasive guiding to programmed ventricular stimulation two-step approach. J Arrhythmia 36:890-898

18. Sabbag A, Glikson M, Suleiman M, Boulos M, Goldenberg I, Beinart R et al (2019) Arrhythmic burden among asymptomatic patients with ischemic cardiomyopathy and an implantable cardioverter-defibrillator. Heart Rhythm 16:813-819

19. Pazhenkottil AP, Buechel RR, Nkoulou R, Ghadri JR, Herzog BA, Husmann L et al (2011) Left ventricular dyssynchrony assessment by phase analysis from gated PET-FDG scans. J Nucl Cardiol 18:920-925

20. Wang L, Wei H-X, Yang M-F, Guo J, Wang J-F, Fang W et al (2013) Phase analysis by gated F-18 FDG PET/CT for left ventricular dyssynchrony assessment: a comparison with gated Tc99m sestamibi SPECT. Ann Nucl Med 27:325-334

Publisher's Note Springer Nature remains neutral with regard to jurisdictional claims in published maps and institutional affiliations. 